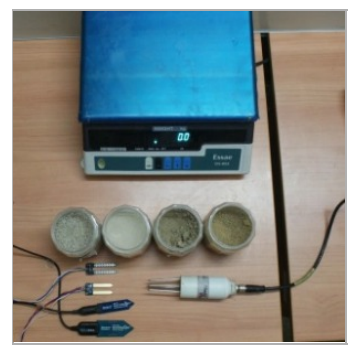

\title{
(3) Laboratory calibration of soil moisture sensors in porous media (repacked soils) V.2
}

Soham Adla ${ }^{1}$, Neeraj Rai ${ }^{2}$, K Sri Harsha ${ }^{2}$, Shivam Tripathi ${ }^{3}$, Markus Disse ${ }^{1}$, Saket Pande ${ }^{4}$

${ }^{1}$ Technische Universität München; ${ }^{2}$ Kritsnam Technologies; ${ }^{3}$ Indian Institute of Technology, Kanpur;

${ }^{4}$ Delft University of Technology

\section{Soham Adla}

Technische Universität München

\section{VERSION 2}

MAR 13, 2019

\section{OPEN ӘA ACCESS}

\section{DOI}

dx.doi.org/10.17504/protocol s.io.swnefde

Protocol Citation: Soham Adla, Neeraj Rai, K Sri Harsha, Shivam Tripathi, Markus Disse, Saket Pande 2019

Laboratory calibration of soil moisture sensors in porous media (repacked soils). protocols.io

rotocols.io.swnefde

License: This is an open access protocol distributed under the terms of the Creative Commons Attribution License, which permits unrestricted use, distribution, and reproduction in any medium, provided the original author and source are credited

Protocol status: Working We use this protocol and it's working

Created: Aug 24, 2018

Last Modified: Mar 13, 2019

PROTOCOL integer ID:

15022

Keywords: Spectrum SM100, Low-cost, soil moisture sensors

\section{Preparation of porous media (repacked soils)}

1 Repacked soils are soil core samples in which the secondary soil structure has been removed (Czarnomski et al., 2005). It has been suggested that for high precision, soil moisture probes should be calibrated using repacked soils (Starr and Paltineanu, 2002).

- Take different types of standard and/or field soils, depending on the possibility of occurrence in the field of application of the sensors to be calibrated. The weight of each soil type should be such that they can fill 5 containers selected for the calibration procedure till about $3 / 4$ th of the container. Container size is given in Step 3: 'Preparation of containers'. The selection of different soil types for calibration should depend on both standardized soil types and locally available soils where the calibrated soil moisture sensors are required to be used. The 4 different soil types chosen in the study are given in Figure 1 and their relevant properties are described in the Table 1. Please note that the soil texture classification is according to the US Department of Agriculture (USDA).

\begin{tabular}{|l|l|l|l|}
\hline Soil Number & Soil Nomenclature & Relative Bulk Density & Soil texture \\
\hline 1 & ISO Grade I sand $(1-2 \mathrm{~mm})$ & 1.82 & Sand \\
\hline 2 & ISO Grade III sand $(0.09-0.5 \mathrm{~mm})$ & 1.588 & Sand
\end{tabular}




\begin{tabular}{|l|l|l|l|}
\hline 3 & $\begin{array}{l}\text { Field soil from experimental site at IIT } \\
\text { Kanpur }\end{array}$ & 1.23 & Silt-Loam \\
\hline 4 & Graded Silt-loam & 1.197 & Silt-Loam \\
\hline
\end{tabular}

- Ensure the removal of the secondary soil structure (macropores, soil aggregates, rocks and large organic material).

- Dry them in air for a sufficiently long time so that any remaining soil moisture is removed. Figure 2 shows the air drying process of the different soils.

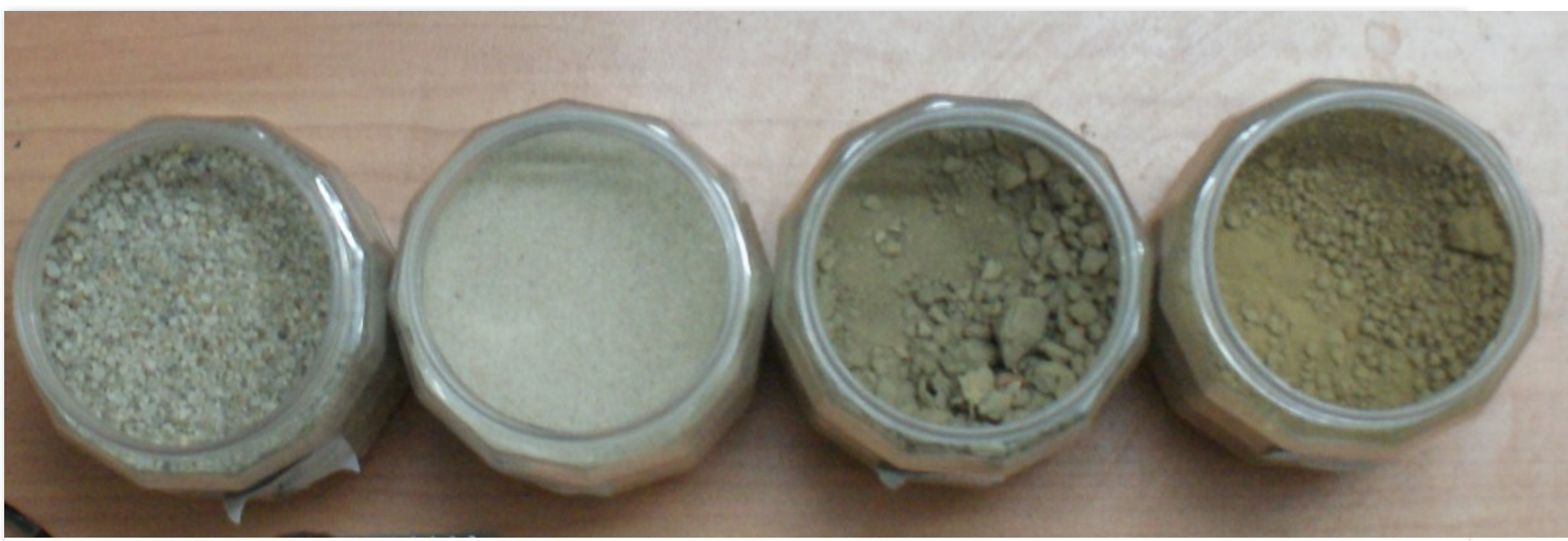

Figure 1: The 4 different soil types chosen for the calibration study. From L to R: Grade I Sand (Indian Standard), Grade III Sand (Indian Standard), Silt-Loam soil from local field, Graded Silt-Loam soil.

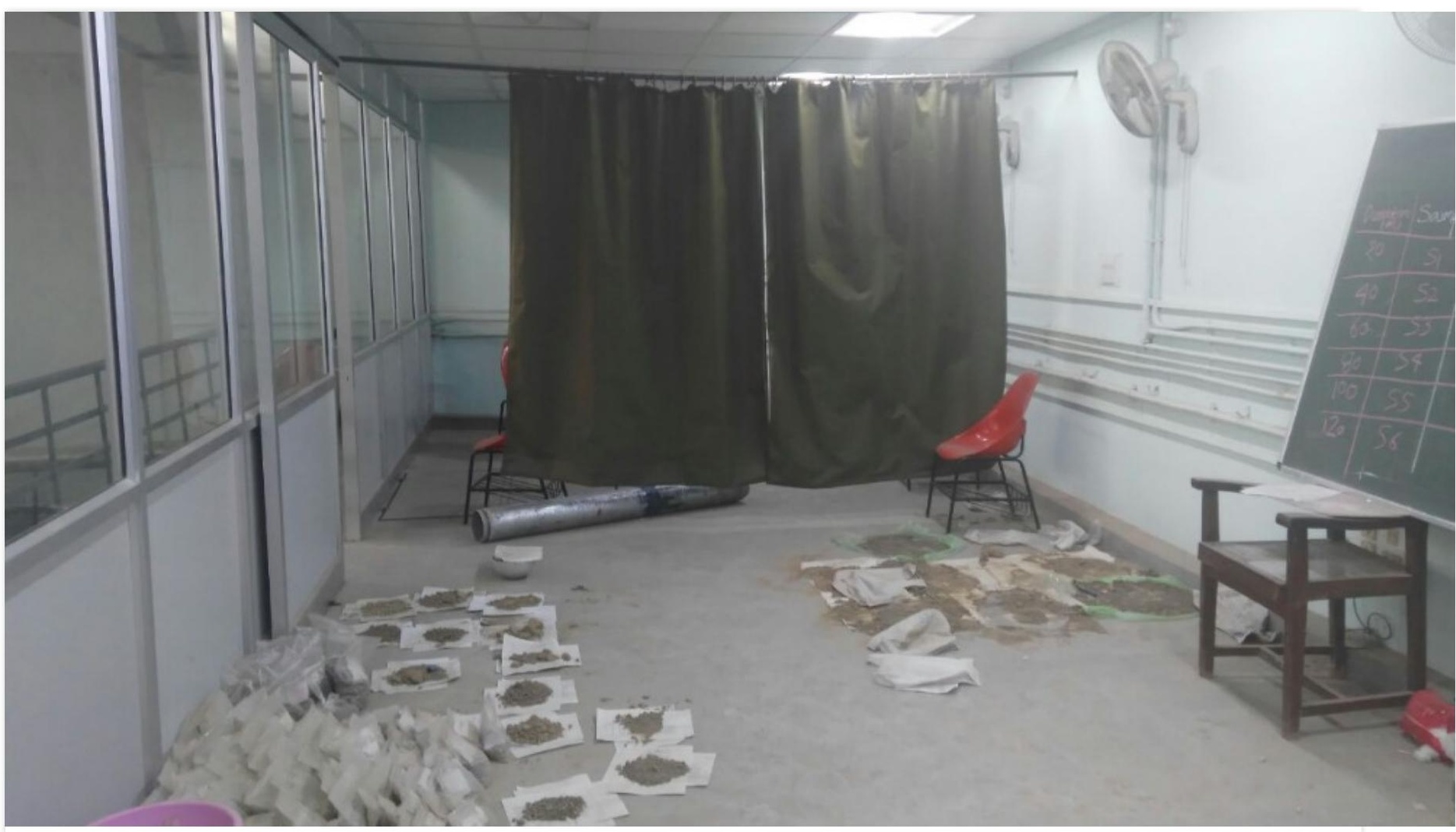

Figure 2: Air drying the different soil samples chosen for the calibration study.

Tools: Tools for removing soil structure (mallet etc.). 


\section{Preparation of containers}

2 This step is based on the standard calibration procedure suggested by Decagon Devices Inc. (Campbell, et al., 2007) applied by Matula et al. (2016).

- Take 5 identical plastic containers for each type of soil to be tested. Container sizes should be such that they are larger than the volume of influence of the soil moisture sensors to be calibrated and also not too large that there is a tendency for internal soil moisture gradients to form. The containers chosen in this case have a diameter of about $10 \mathrm{~cm}$ and a height of about $15 \mathrm{~cm}$. This is larger than the volumes of influence which can be estimated using the available literature, given below:

\begin{tabular}{|c|c|c|}
\hline Soil moisture sensor (and make) & Volume of influence & Link to reference \\
\hline $\begin{array}{l}\text { WaterScout SM } 100 \text { Sensor (Spectrum Technologies, } \\
\text { Inc.) }\end{array}$ & $\begin{array}{l}\text { Sensing area }=6 \mathrm{~cm} \times 2 \\
\mathrm{~cm}\end{array}$ & $\begin{array}{l}\text { Spectrum Technologies (2014). Retrieved from } \\
\text { https://www.specmeters.com/weather-monitoring/sensors- } \\
\text { and-accessories/sensors/soil-moisture-sensors/sm100/ }\end{array}$ \\
\hline $\begin{array}{l}\text { WaterScout SMEC } 300 \text { Sensor (Spectrum } \\
\text { Technologies, Inc.) }\end{array}$ & $\begin{array}{l}\text { Sensing area }=5.715 \\
\mathrm{~cm} \times 1.905 \mathrm{~cm}\end{array}$ & $\begin{array}{l}\text { Spectrum Technologies (2015). Retrieved from } \\
\text { https://www.specmeters.com/weather-monitoring/sensors- } \\
\text { and-accessories/sensors/soil-moisture-sensors/smec300/ }\end{array}$ \\
\hline $\begin{array}{l}\text { KitsGuru Soil Moisture Sensor Module (Generic, } \\
\text { distributed in India through Cloudtail India) }\end{array}$ & $\begin{array}{l}\text { Not specified } \\
\text { (Sensing Probe } \\
\text { Dimensions }=6 \mathrm{~cm} \times 3 \\
\mathrm{~cm} \text { ) }\end{array}$ & $\begin{array}{l}\text { https://www.amazon.in/dp/B00XU8MJ4E/ref=pe_1909141_ } \\
\text { 77686011_tnp_emaildp_1 }\end{array}$ \\
\hline $\begin{array}{l}\text { SEN6 Soil Hygrometer Detection Module Soil Moisture } \\
\text { Sensor (Robodo Electronics) }\end{array}$ & $\begin{array}{l}\text { (Sensing Probe } \\
\text { Dimensions }=5 \mathrm{~cm} \times 3 \\
\mathrm{~cm} \text { ) }\end{array}$ & $\begin{array}{l}\text { https://www.amazon.in/Robodo-Electronics-SEN6- } \\
\text { Hygrometer-Detection/dp/B0787HGY18/ref=sr_1_15? } \\
\text { s=industrial\&ie=UTF8\&q }\end{array}$ \\
\hline
\end{tabular}

- Label each container using a convenient approach, with 5 containers for each soil type. This specific calibration study uses simple incremental numbering - container 1-5 for soil type 1, container 6-10 for soil type 2, container 11-15 for soil type 3 and container 16-20 for soil type 4 (Figure 3 illustrates all the soil samples and their respective containers used in the study). Weigh each container and make a record. Please check the 'Containers' sheet of an example file 'Template_tables.xls' (given in Step 7: 'Data tabulation and calibration relationships for sensors') that can be used as a template.

- Add a similar amount of soil to each of the five containers assigned for that particular soil type.

- Weigh each container again with the soil inside and make a record. Please check the 'Containers' sheet of an example file 'Template_tables.x/s' (given in Step 7: 'Data tabulation and calibration relationships for sensors') that can be used as a template.

- Calculate the volume of the soil contained in each container with the formula. Please check the 'Containers' sheet of an example file 'Template_tables.x/s' (given in Step 7: 'Data tabulation and calibration relationships for sensors') that can be used as a template.

- Add water in each of the 5 containers for each soil type so that a range of VWC can be covered for each soil type, from very dry (corresponding to VWC $=0 \%$ ) to very wet (approaching saturated VWC which will depend on the soil type). Measure the added volume (using a measuring device like a graduated cylinder) and make a record of the same. Please check the 'Containers' sheet of an example file 'Template_tables.xIs' (given in Step 7: 'Data tabulation and calibration relationships for sensors') that can be used as a template. One suggestion is to fill the chronologically last container of each soil type with enough (measured water) water resulting in a saturated soil + water slurry and subsequently adding $25 \%$, $50 \%$ and $75 \%$ of the added amount in the last (near saturated container) to the $2^{\text {nd }}, 3^{\text {rd }}$ and $4^{\text {th }}$ container of that soil type.

- Compute the initial VWC of each container using the following formula, which is also given in 'Template_tables.x/s' (given in Step 7: 'Data tabulation and calibration relationships for sensors'):

VWC(initial) [\%] = 100 * (Volume of water added [cc]) / (Weight of soil [g] / Bulk density of soil [g/cc])

\section{Preparation of soil moisture sensors for calibration}

3 Preparation of the soil moisture sensors depends on whether a data logger/reader has already been procured, or whether they are based on open source electronics tools. In this specific study, the Arduino UNO microcontroller board (developed by Arduino) was used.

Sensors with already available data logger/reader: As an example, the SM100 sensor (Spectrum Technologies, 2014) can be calibrated back to factory settings using a two point calibration method (VWC in air = 0\%. VWC in distilled water $=$ around $55 \%$ ). If the SM100 sensor has a ' $3 / 14$ ' code printed on the front, it can be re-calibrated to the factory setting. The soil moisture circuit of the SMEC 300 sensor cannot be recalibrated back to the factory setting. The following is the recalibration method for the SM100 sensor.

- Use the Spectrum Field Scout Soil Sensor Reader and attach the SM100 sensor to it.

- Place the sensor in distilled water, and press the 'CAL EC?'. A long press on the " button will calibrate the sensor to its factory settings. More details are given in the external link (https://www.specmeters.com/assets/1/22/6466_Soil_Moisture_Sensor_Reader4.pdf)

Sensors with open source data: The KitsGuru Soil Moisture sensor and the Robodo Electronics' SEN5 Soil Hygrometer sensor are available off the market and do not have default factory settintgs. Hence, there is a need to only connect them via open source electronics (such as the Arduino UNO microcontroller board) to ease the process of reading data from a computer screen while calibrating the sensors.

- Follow the methodology given in the attached document ('Arduino_reader_code.ino') to develop a data reader for these sensors. 


\section{Simultaneous sensor and gravimetric measurements}

4 A template for the data entry corresponding to all the sensor measurements during calibration is given in the 'Calibration' sheet of the example file

'Template_tables.xls' (given in Step 7: 'Data tabulation and calibration relationships for sensors').

- Properly mix the soil + water mixture inside the first container of the first soil type with an appropriate tool ensuring that there is minimal loss of soil due to the insertion of the tool. This is done to prevent the development of soil moisture gradients within the container. As a general rule, mix any soil carefully before taking a reading, ensuring minimal loss of weight due to accidentally removed soil particles.

- Weigh the container (container + soil + water). Make a record of the weight. This leads to a measure of the actual weighted VWC inside the container.

- Insert a sensor with a higher accuracy, which can act as a secondary standard for calibration. In this calibration setup, the more accurate impedence based soil moisture sensor, ThetaProbe ML2x (Delta-T Devices, 1999) was used to get a sensor based accurate measurement of VWC. Make a record of the measured VWC. Remove the secondary standard sensor.

- Insert the soil moisture sensor (which needs to be calibrated) into the soil and take a reading of the measured raw sensor output as well as the factory calibrated VWC value (if it exists). Make a record of the reading.

- Wait for 30 minutes and repeat the measurement and record the reading. For automated recording, multiple readings can be averaged and a standard deviation/error can be computed, as is given in 'Template_Tables.xls'. The interval between readings is recommended due to the process of stabilization of the soil + water matrix around the sensor material. Also, it leads to an estimation of sensor precision due to the multiple measurements with the same conditions of measurement. The duration (30 minutes) is an indicative recommendation based on the time to stabilize soil moisture readings in local soils for the instruments used, and may vary for different soil + sensor combinations.

- Remove the soil moisture sensor being calibrated, re-insert the impedence based ThetaProbe soil moisture sensor, take another reading and make a record of it. This additional reading averages out pre-and post-measurement conditions of soil packing which may have changed due to the insertion of the instruments. Remove the sensor.

- Wipe each sensor after removal from a container with a brush to return the soil to the container. This is done to prevent any loss of soil, and to ensure that any reductions in weight are only due to the loss of evaporated water and not soil.

- Weigh the container again. Weights are taken both before and after all the measurements to average out any minor changes in weight from losses in water.

Tools: appropriate tool for mixing soil + water mixture

\section{Repeated measurements for all sensors}

5 Repeat the procedure given in Step 3 and Step 4 for each soil type selected for the calibration. The end experiment should be such that each sensor to be calibrated has 5 point measurements for each soil type (using five nearly identical soil sample replicates) which can be then used for developing calibration equations. 


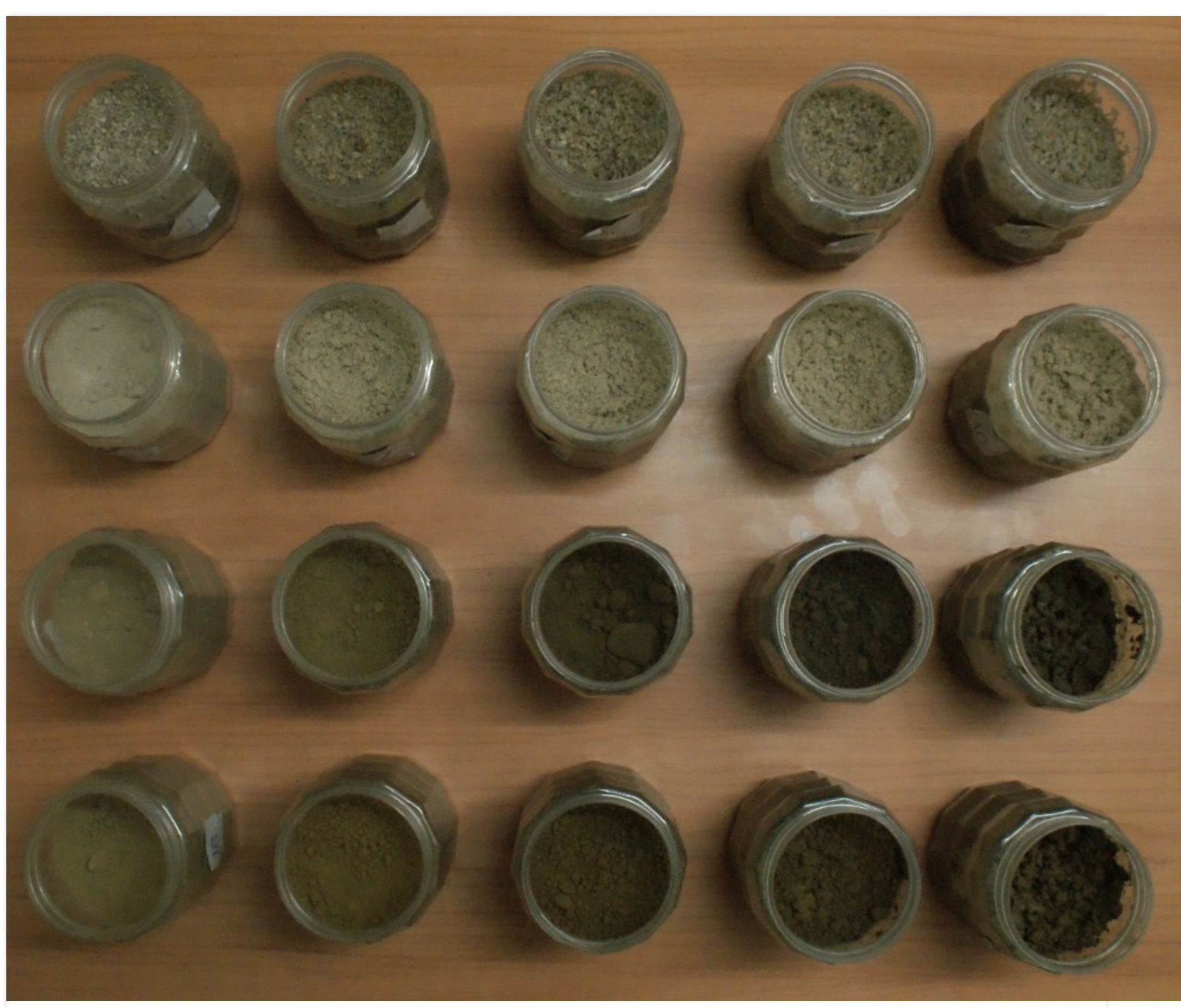

Figure 4: The 4 soil types used in the specific calibration study. Row ' $i$ ' (1 to 4) corresponds to Soil Type ' $i$ ' (1 to 4) and the VWC is increasing from the first column (driest) to the last column (wettest).

\section{Data tabulation and calibration relationships for sensors}

6 Tabulate all the data to generate calibration curves. A template for the data entry for all data during the entire calibration process is given in the example file 'Template_Tables.xls'. It has data entry templates for the following:

- Experiment to measure bulk density of the repacked soils.

- Preparation of containers.

- Calibration experiment with simultaneous sensor and weight measurements.

Selecting the actual VWC values as the dependent variable, and the measured VWC (from the different sensors) as independent variable, run a regression using an appropriate software (MS Excel, STATA, SPSS, R), which would then depend on the user based decisions on type of regression chosen (linear, non-linear etc.).

Template_Tables.xlsx

\section{References}

- Czarnomski, N. M., Moore, G. W., Pypker, T. G., Licata, J., \& Bond, B. J. (2005). Precision and accuracy of three alternative instruments for measuring soil water content in two forest soils of the Pacific Northwest. Canadian Journal of Forest Research, 35(8), 1867-1876. 
- Starr, J.L and Paltineanu, I.C. (2002). Methods for measurement of soil water content: capacitance devices. In Methods of soil analysis. Part 4. Physical methods. Edited by J.H. Dane and G.C. Topp. Soil Science Society of America, Madison, Wis. pp. 463-474.

- Campbell, C.S.; CCobos, D.R.; Bissey, L.L. (2007). Calibration and Evaluation of an Improved Low-Cost Soil Moisture Sensor, Application Note 800-755-2751; Decagon Devices, Inc.: Pullman, WA, USA. p. 13

- Delta-T Devices (1999). ThetaProbe soil moisture sensor type ML2x user manual. Delta-T Devices, Cambridge, UK.

- Matula, S., Bát'ková, K., \& Legese, W. L. (2016). Laboratory performance of five selected soil moisture sensors applying factory and own calibration equations for two soil media of different bulk density and salinity levels. Sensors, 16(11), 1912.

- Spectrum Technologies (2014). Waterscout SM100 Soil Moisture Sensor Product Manual. Spectrum Technologies, Inc., 12350 S. Industrial Dr. East Plainfield, IL (USA). Retrieved from https://www.specmeters.com/assets/1/22/6460_SM1002.pdf

- Spectrum Technologies (2015). Waterscout SMEC 300 SoilMoisture Sensor Product Manual. Spectrum Technologies, 430 Inc., 3600 Thayer Court, Aurora, IL (USA). Retrieved from https://www.specmeters.com/assets/1/22/6470_SMEC3004.pdf 